\title{
Precision requirement of the photofission cross section for the nondestructive assay
}

\author{
Rei Kimura ${ }^{a}$, Hiroshi Sagara, and Satoshi Chiba \\ Tokyo Institute of Technology, 2-12-2 Ookayama Meguro-ku Tokyo, Japan
}

\begin{abstract}
Principle of the new NDA technique based on the photofission reaction rate ratio (PFRR) has been developed by Kimura et al for measurement of uranium enrichment by using the only relative measured counts of neutron produced by photofission reactions of ${ }^{235} \mathrm{U}$ and ${ }^{238} \mathrm{U}$ at different specific incident photon energies. In the past analysis, no attentions have been paid for relatively large uncertainty of photonuclear cross section of special nuclear materials around $10 \%$. In the present paper, quantitative analysis was performed to reveal the impact of photonuclear cross section uncertainty to predicted value of the uranium enrichment by the PFRR methodology. And also, the requirement of photofission cross section precision was evaluated as less than $3 \%$, to satisfy the uncertainty of PFRR methodology to within 5\%.
\end{abstract}

\section{Introduction}

The nondestructive assay (NDA) techniques for quantifying special nuclear materials (SNMs) have been developed by many organizations and some of which have been successfully applied to uranium enrichment measurement [1-9]. One of the recent projects is Next Generation Safeguards Initiative in the United States which has been examined in a spent fuel NDA technique [2]. The other challenge of the NDA technique for quantification or even detection of SNMs in unknown forms, such as unknown waste, debris or concealed and shielded highly enriched uranium in containers, these have some technical difficulty as follows [10];

(1) Few self-generated neutron or photon emissions because of shielding

(2) Difficulty of measurement because of intensive gamma-ray backgrounds

(3) Low measurement reliability due to impurities and unknown information.

Recently, the development of the compact and quasimonochromatic photon (X-ray) source generator has proceeded, which is expected to be realized as portable photon generator device with higher energy than the photonuclear threshold energy [11-14]. Its application is expected to be one of the NDA techniques.

A new NDA technique is aimed for uranium enrichment measurement, characterized by mathematical process which represents the correlation of the target enrichment and relative measured counts of neutron produced by the photofission reactions of ${ }^{235} \mathrm{U}$ and ${ }^{238} \mathrm{U}$ at different specific incident photon energies of $6 \mathrm{MeV}$ and $11 \mathrm{MeV}$. Principle of the nuclear material isotopic composition measurement method based on the

a e-mail: author@e-mail.org photofission reaction rate ratio (PFRR) was validated by small scale numerical simulation with good reproducibility of within $2 \%$ difference of predicted uranium enrichment and reported by Kimura et al. [10]. However, cross sections of the photonuclear reaction of interested nuclides relating to PERR have, in general, around $10 \%$ uncertainty, which may lead the huge impact to the accuracy of uranium enrichment measurement by the PFRR methodology. In the present paper, quantitative analysis was performed to reveal the impact of photonuclear cross section uncertainty to predicted value of the uranium enrichment by the PFRR methodology. And also, the requirement of photonuclear cross section precision was evaluated

\section{Principle of the NDA technique based on the Photofission reaction rate ratio}

The PFRR methodology mechanism is based on the difference of photonuclear cross section of different nuclides and different incident photon energies, these functions of the incident photon energies for the typical fertile and fissile nuclides of ENDF/B-VII.1 are shown in Fig. 1 [14]. These differences of cross sections make the differences of neutron production rate at the target of SNMs, for example, as shown in Fig. 2 [10].

The neutron production rates shown in Fig. 2 include the $(\gamma, \mathrm{n}),(\gamma, 2 \mathrm{n}),(\gamma$, fission $)$, and other neutron production reactions. In case of the maximum incident photon energy is under $11.27 \mathrm{MeV}$ as threshold energy of $(\gamma, 2 n)$ reaction at ${ }^{238} \mathrm{U}$ and ${ }^{235} \mathrm{U}$ target, $(\gamma$, fission) counts can be extracted from the neutron counts by coincidence counting. In the PFRR methodology, the information of photofission reactions is utilized to improve the precision by the simplified mathematical process as removal of other reactions from the equation.

The photofission reaction rate $R_{i}$ ( $i$ represents the specific incident photon energy spectrum) is described

(C) The Authors, published by EDP Sciences. This is an Open Access article distributed under the terms of the Creative Commons Attribution License 4.0 (http://creativecommons.org/licenses/by/4.0/). 
by Eq. (1),

$$
R_{i}=\int \phi_{i}(E) \sum_{n u c}\left(N_{n u c} \sigma_{f, n u c}(E)\right) d E,
$$

where $E$ is the photon energy, $\phi_{i}(E)$ is the photon flux, $N_{n u c}$ and $\sigma_{f, n u c}(E)$ are number density and microscopic photofission cross section of nuclide nuc. In addition, parameters $i$ and $n u c$ are defined as $1,2,3 \ldots n$ and $I, I I, I I I \cdots n$. Further, $A_{i, n u c}$ is defined as $A_{i, n u c}=$ $\int \phi_{i}(E) \sigma_{f, n u c}(E) d E$ and $R_{1} \sim R_{n-1}$ are divided by $R_{n}$, Eq. (1) for each $i$ and $n u c$ can be transformed as Eq. (2), where $A_{i, n u c}$ is known. The PFRR methodology requires the measurement value of the photofission reaction rate ratio $R_{i} / R_{n}$ in order to calculate $N_{n u c} / N_{n}$. The isotopic composition IC of nuclide nuc is calculated from $N_{n u c} / N_{n}$ and Eq. (3).

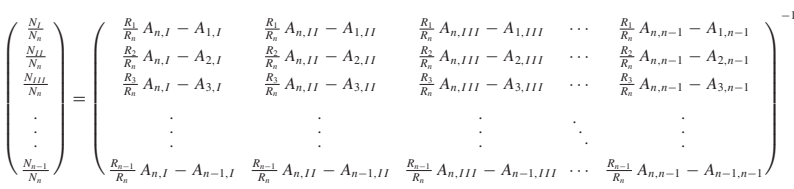

$$
\begin{aligned}
& \times\left(\begin{array}{c}
A_{1, n}-\frac{R_{R}}{R_{R}} A_{n, n} \\
A_{2, n}-\frac{R_{R}}{R_{R}} A_{n, n} \\
A_{3, n}-\frac{R_{R}}{R_{R}} A_{n, n} \\
\vdots \\
A_{n-1, n}-\frac{R_{n-1}}{R_{n}} A_{n, n}
\end{array}\right) \\
& I C_{n u c}=\frac{N_{n u c}}{N_{I}+N_{I I}+N_{I I I}+\cdots+N_{n}} \\
& =\frac{\frac{N_{n u c}}{N_{n}}}{\frac{N_{I}}{N_{n}}+\frac{N_{I I}}{N_{n}}+\frac{N_{I I I}}{N_{n}}+\cdots+1}
\end{aligned}
$$

Hence, the PFRR methodology induces the isotopic composition by only measuring relative value of the photofission reaction [10].

\section{Calculation model and methodology}

MCNP6 as a Monte Carlo code and ENDF/B-VII.1 as an evaluated nuclear data library were used for simulating the photonuclear reaction in the target $[14,15]$. Figure 3 shows the calculation model of the present study. In this model, the photon beam is assumed to be injected to the center of the thin target. This target consists of metallic uranium $\left({ }^{235} \mathrm{U}\right.$ and ${ }^{238} \mathrm{U},{ }^{235} \mathrm{U}$ enrichment is $\left.5-90 \%\right)$ which density is $19.1 \mathrm{~g} / \mathrm{cm} 3$.

Incident photons from the pencil beam $\left(10^{8}\right.$ histories in this study) cause the photofission reaction at the target. The fission reaction which occurred at the target is tallied as " $R_{i}$ " of Eq. (2). This fission reaction include ( $\gamma$, fission) and (n, fission) because signal of ( $\gamma$, fission) and ( $n$, fission) cannot be separated in the actual measurement by coincidence counting.

The error propagation formula of predicted ${ }^{235} \mathrm{U}$ enrichment in the ${ }^{235} \mathrm{U}-{ }^{238} \mathrm{U}$ system was derived as Eq. (4),

$$
\varepsilon=\frac{1}{\left(N_{U 235 / N_{U 238}}+1\right)^{2}} \sqrt{\left(\frac{1}{M_{B}} \varepsilon_{A}\right)^{2}+\left(\frac{M_{A}}{M_{B}^{2}} \varepsilon_{B}\right)^{2}}
$$

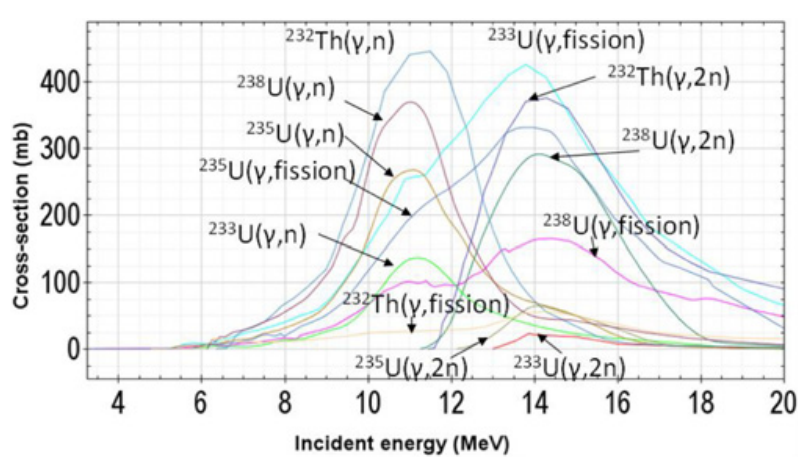

Figure 1. Photonuclear reaction cross sections versus the incident photon energy. The cross section of each nuclides and reactions are written as "nuclides(reaction)" [10,14].

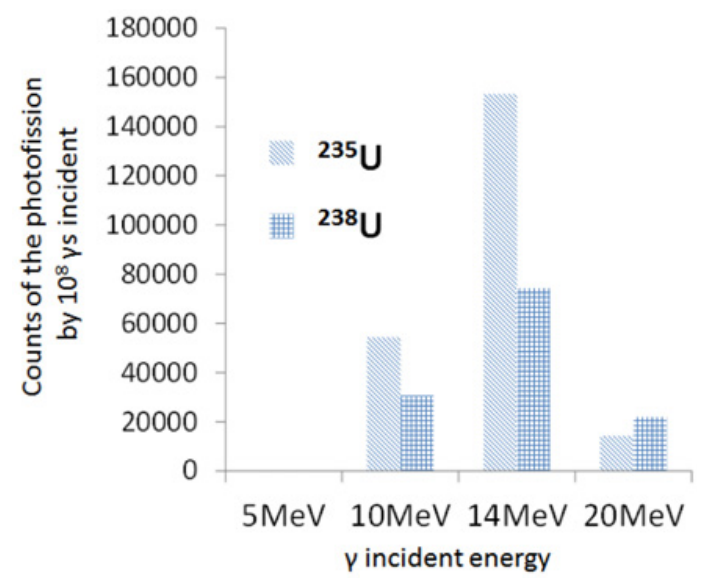

Figure 2. Difference in the neutron production for different photon energies and nuclides [10].

where, $N_{U 235} / N_{U 238}$ and $R_{\text {ratio }}$ was $N_{\text {nuc }} / N_{n}$ and $R_{i} / R_{n}$ of Eq. (2), $\varepsilon_{0,238 U}$ and $\varepsilon_{0,235 U}$ were relative error of the photofission cross section of ${ }^{238} \mathrm{U}$ and ${ }^{235} \mathrm{U}$. Other parameters in the Eq. (4) were described as follows:

$$
\begin{aligned}
& M_{A}=\left(A_{238 U, n}-R_{\text {ratio }} A_{238 U, i}\right) \\
& M_{B}=\left(R_{\text {ratio }} A_{235 U, i}-A_{235 U, n}\right) \\
& \varepsilon_{A}=\sqrt{\left(\varepsilon_{0,238 U} A_{238 U, n}\right)^{2}+\left(\varepsilon_{0,238 U} R_{\text {ratio }} A_{238 U, i}\right)^{2}} \\
& \varepsilon_{B}=\sqrt{\left(\varepsilon_{0,235 U} R_{\text {ratio }} A_{235 U, i}\right)^{2}+\left(\varepsilon_{0,235 U} A_{235 U, n}\right)^{2}} \\
& A_{238 U, i}=\int \sigma_{f, 238 U}(E) \phi_{i}(E), \\
& A_{238 U, n}=\int \sigma_{f, 238 U}(E) \phi_{n}(E) \\
& A_{235 U, i}=\int \sigma_{f, 235 U}(E) \phi_{i}(E), \\
& A_{235 U, n}=\int \sigma_{f, 235 U}(E) \phi_{n}(E) .
\end{aligned}
$$

\section{Results and discussion}

\subsection{Estimation of the ${ }^{235} \mathrm{U}$ enrichment based on the PFRR method}

The results of the ${ }^{235} \mathrm{U}$ enrichment prediction by PFRR method was shown in Fig. 4. The incident photon energies are 11 and $6 \mathrm{MeV}$ that has the Gaussian shaped energy distribution $(\sigma=0.5 \mathrm{MeV})$ [10]. As shown in this figure, the present method showed good reproducibility of ${ }^{235} \mathrm{U}$ enrichment, the principle of PFRR methodology was 


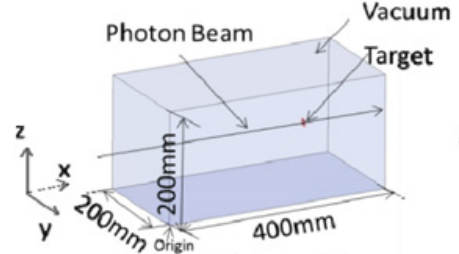

(a) Whole model

Figure 3. Calculation model on the MCNP code.

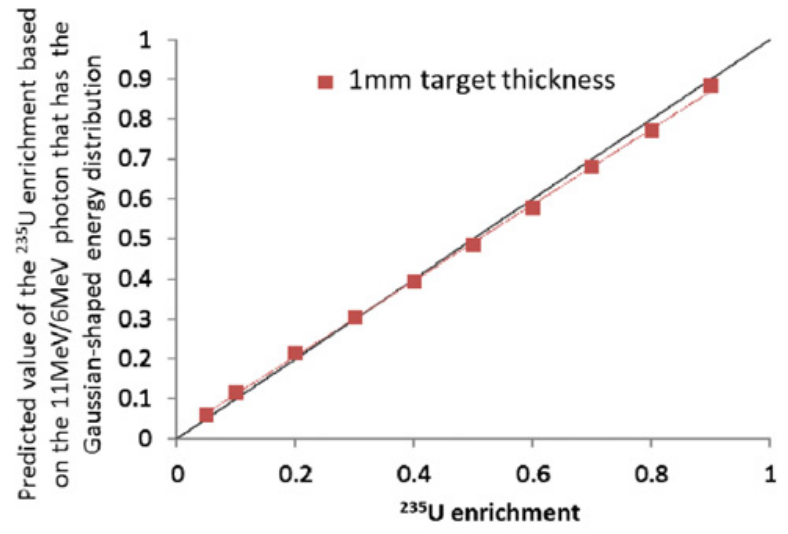

Figure 4. The predicted value of the ${ }^{235} \mathrm{U}$ enrichment based on the PFRR due the $11 \mathrm{MeV} / 6 \mathrm{MeV}$ incident photon that has the Gaussian shaped energy distribution [10].

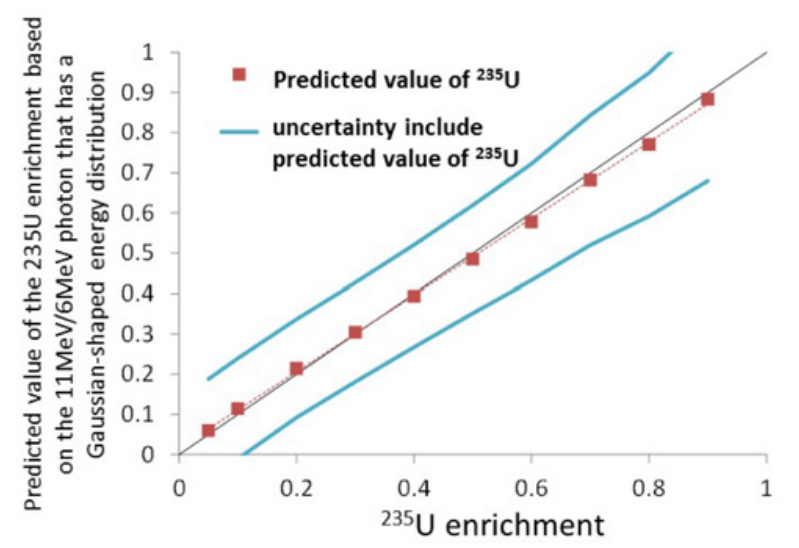

Figure 5. The predicted value of ${ }^{235} \mathrm{U}$ enrichment and its uncertainty with $10 \%$ cross section uncertainty.

shown to be applicable to predict of SNM isotopic composition.

\subsection{Implication of the photofission cross section uncertainty}

Assuming $10 \%$ uncertainty of the photofission cross section of ${ }^{235} \mathrm{U}$ and ${ }^{238} \mathrm{U}$, predicted value of the ${ }^{235} \mathrm{U}$ enrichment had a $13 \%$ uncertainty at $20 \%$ enrichment as shown in Fig. 5.

This uncertainty was reduced by decreasing of the cross section uncertainty. In addition, as shown in Fig. 6, $3 \%$ or less cross section uncertainty was required to reduce the uncertainty of predicted value of the ${ }^{235} \mathrm{U}$ enrichment to less than $5 \%$.

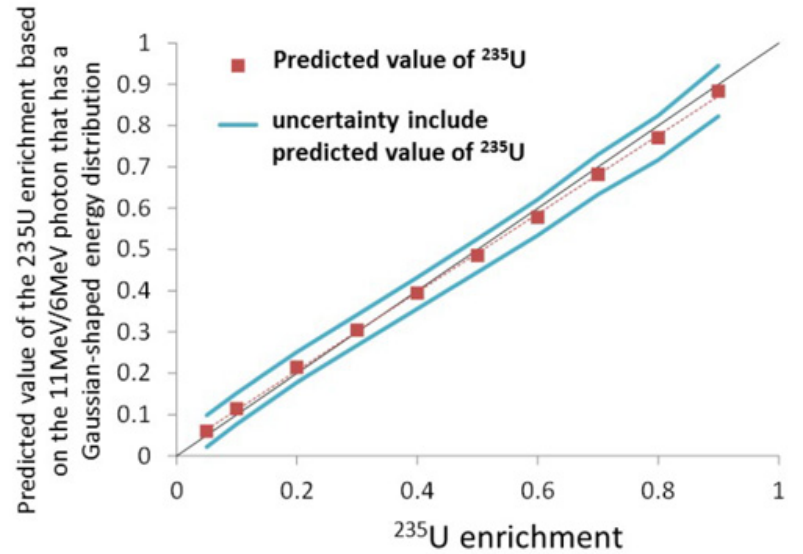

Figure 6. The predicted value of ${ }^{235} \mathrm{U}$ enrichment and its uncertainty with $3 \%$ cross section uncertainty.

\section{Conclusion}

The effect of the photofission cross section uncertainty to the predicted value of the ${ }^{235} \mathrm{U}$ in the PFRR methodology was evaluated. This uncertainty was required to be $3 \%$ or less to keep less than $5 \%$ uncertainty of the predicted value of the ${ }^{235} \mathrm{U}$ enrichment.

However, the current photonuclear cross section data of nuclear materials, namely, uranium and plutonium nuclides have generally $10 \%$ or more cross section uncertainty. Therefore, the photonuclear cross sections, especially photofission cross sections of uranium and plutonium, of these nuclides are strongly desired of precision improvement for uncertainty reduction of the PFRR methodology.

\section{References}

[1] S. Iso, I. Kobayashi, S. Masuda, et al., Proceedings of the $32^{\text {nd }}$ Annual Meeting of INMM Japan Chapter, Status of the development of safeguards for large scale MOX fuel fabrication plant (2011)

[2] W.S Charlton, M.A Humphrey, J Nucl Mat Maneg, External review of the next generation safeguards initiative's spent fuel nondestructive assay project 40, 18 (2012)

[3] H. Ohgaki, T. Kii, K. Masuda, et al., Procceding of IEEE HST, Conceptual design of a nuclear material detection system based on the neutron/gamma-ray hybrid approach, 525-529 (2010)

[4] R. Hajima, T. Shizuma, T. Hayakawa, et al., Proceedings of IPAC, Compact gamma-ray source for non-destructive detection of nuclear material in cargo, 3663-3665 (2011)

[5] R. Hajima, T. Hayakawa, N. Kikuzawa, et al., J. Nucl. Sci. Technol. Proposal of nondestructive radionuclide assay using a high-flux gamma-ray source and nuclear resonance florescence 45, 441 (2008)

[6] M. Seya, H. Harada, F. Kitatani, et al., Paper presented at: INMM 54 ${ }^{\text {th }}$ Annual Meeting, Development of basic NDA technologies for nuclear material accountancy of debris of melted fuel formed in severe accidents (2013)

[7] M. Seya, H. Harada, F. Kitatani, et al., Proceedings of $35^{\text {th }}$ ESARDA Annual Meeting, Promising NDA technologies for material accountancy of nuclear 
material in debris of melted fuel of FukushimaDaiichi NPP (2013)

[8] H. Sagara, H. Tomikawa, M. Watahiki, et al., J. Nucl. Sci. Technol. Feasibility study of passive gamma spectrometry of molten core material from Fukushima Daiichi Nuclear Power Station unit 1, 2, and 3 cores for special nuclear material accountancy -low-volatile FP and special nuclear material inventory analysis and fundamental characteristics of gamma-rays from fuel debris 51, 1 (2014)

[9] Y. Nauchi, H. Ohta, H. Unesaki, et al., J. Nucl. Sci. Technol. Concept of capture credit based on neutron-induced gamma ray spectroscopy 52, 10741083 (2015)

[10] R. Kimura, H. Sagara, S. Chiba, J. Nucl. Sci. Technol. Principle validation of nuclear fuel material isotopic composition measurement method based on photofission reactions 53, 1978-1987 (2016)
[11] S. Chen, D. Powers, I. Ghebregziabher, et al., Phys. Rev. Lett. MeV-energy X rays from inverse Compton scattering with Laser-wakefield accelerated electrons 110, 155003 (2013)

[12] S. Miyamoto, Y. Asano, S. Amano, et al., Rad. Meas. Laser Compton back-scattering gamma-ray beamline on NewSUBARU 41, S179 (2006)

[13] S. Boucher, P. Frigola, A. Murokh, et al., Nuc. Inst. Methods Phys. Res. A, Inverse Compton scattering gamma ray source 608, S54-56 (2009)

[14] M.B Chadwick, M. Herman, P. Oblozinsky, et al., Nucl. Data Sheets, ENDF/B-VII.1 nuclear data for science and technology: cross sections, covariances, fission product yields and decay data 112, 2887-2996 82011)

[15] T. Goorley, M. James, T. Booth, et al., Nucl. Technol. Initial MCNP6 release overview $\mathbf{1 8 0}$, 298-315 (2012) 